\title{
Prevalência do refluxo na veia safena parva em varizes primárias não complicadas dos membros inferiores pelo eco-D oppler colorido
}

\author{
Prevalence of short saphenous vein reflux \\ in primary uncomplicated varicose veins by $D$ oppler ultrasonography
}

\author{
Fabio Secchi ${ }^{1}$, M arcio M iyamotto ${ }^{2}$, G raciliano J osé França ${ }^{3}$, Aguinaldo de 0 liveira4, \\ Enrique Antônio Vidal', Jorge R. Ribas T imi' ${ }^{6}$, Ricardo C. Rocha M oreira7
}

\begin{abstract}
O bjetivo: Determinar a prevalência de refluxo venoso na veia safena parva em membros inferiores com varizes primárias não complicadas pelo eco-D oppler colorido.

M étodo: No período de 18 meses, 1.953 pacientes foram submetidos ao eco-D oppler colorido de membros inferiores por doença ve nosa. D estes, 1.631 com varizes primárias não complicadas foram selecionados para esta análise, sendo que 1.383 eram do sexo feminino $(84,79 \%)$ e $248(15,21 \%)$ do sexo masculino. A média de idade dos pacientes foi de $42,89( \pm 0,48)$ anos, variando de 13 a 85 anos. D os 1.631 pacientes, 1.323 foram submetidos a exame bilateral e 308 a exame unilateral, totalizando 2.954 membros inferiores com varizes primárias não complicadas avaliados. D esse total, 1.461 eram membros inferiores direitos e 1.493, esquerdos. Todos os exames foram realizados seguindo o mesmo protocolo.

Resultado: Dos 2.954 membros inferiores avaliados, 372 $(12,59 \%)$ apresentaram refluxo em veia safena parva. A prevalência nos homens foi de $14,08 \%$ e, nas mulheres, de $12,35 \%$. 0 refluxo da safena parva foi maior no membro inferior esquerdo (13.13\%) do que no direito (12,05\%). A prevalência do refluxo foi significativamente maior nos pacientes acima de 60 anos.

Conclusão: 0 refluxo da veia safena parva é relativamente comum, e sua pesquisa deve ser sempre real izada em pacientes com varizes primárias de membros inferiores.
\end{abstract}

Palavras-chave: Veia safena, varizes, ultra-sonografia.

\begin{abstract}
O bjective: To determine the prevalence of small saphenous vein reflux (SSVR) in patients with uncomplicated varicose veins, using color-flow D oppler ultrasonography.

Method: 0 ver an 18-month period, a total of 1,953 patients underwent color-flow Doppler ultrasonography for evaluation of venous disease. 0 ut of the total, 1,631 patients with primary uncomplicated varicose veins were selected for this study: 1,383 $(84.79 \%)$ patients were female and $248(15.21 \%)$ were male. M ean age was $42.9( \pm 0.48)$ years, ranging from 13 to 85 years. Of the 1,631 patients, 1,323 underwent bilateral examination and 308 unilateral examination, for a total of 2,954 lower limbs with primary uncomplicated varicose veins studied. The number of right lower limbs studied was 1,461 and the number of left lower limbs was 1,493. All exams were carried out using the same protocol.

Result: 0 ut of the total number of 2,954 limbs, SSVR was detected 372 (12.59\%) limbs. Prevalence of SSVR in males was $14.08 \%$ and in females was $12.35 \%$. SSVR was detected in the right lower limb in $12.05 \%$ and in left lower limb in $13.13 \%$ of the cases. SSVR was significantly more common in patients older than 60 years.

Conclusion: SSVR is relatively common and its presence should always be investigated in patients with primary lower limb varicose veins.
\end{abstract}

Key words: Saphenous vein, varicose veins, ultrasonography.

1. Cirurgião vascular, Dourados, MS. Mestre em Clínica Cirúrgica, Universidade Federal do Paraná (UFPR), Curitiba, PR.

2. Cirurgião vascular e endovascular, Serviço de Cirurgia Vascular Prof. Dr. Elias Abrão, Hospital Universitário Cajuru, Pontifícia Universidade Católica de Curitiba, Curitiba, PR. Mestrando em Clínica Cirúrgica, UFPR, Curitiba, PR.

3. Ecografista vascular, Clínica Eco-Doppler Colorido e Hospital das Clínicas da UFPR, Curitiba, PR. Mestre em Clínica Cirúrgica, UFPR, Curitiba, PR. Certificado de atuação na área de ecografia vascular com Doppler, SBACV/CBR.

4. Ecografista vascular, Clínica Eco-Doppler Colorido, Curitiba, PR. Mestre em Clínica Cirúrgica, UFPR, Curitiba, PR. Certificado de atuação na área de ecografia vascular com Doppler, SBACV/CBR

5. Ecografista vascular, Clínica Eco-Doppler Colorido, Curitiba, PR. Certificado de atuação na área de ecografia vascular com Doppler, SBACV/CBR.

6. Cirurgião vascular, Serviço de Cirurgia Vascular Prof. Dr. Elias Abrão, Curitiba, PR. Professor adjunto, UFPR, Curitiba, PR. Mestre e Doutor em Clínica Cirúrgica, UFPR, Curitiba, PR.

7. Chefe do Serviço de Cirurgia Vascular Prof. Dr. Elias Abrão, Curitiba, PR. Mestre e Doutor em Clínica Cirúrgica, UFPR, Curitiba, PR.

Trabalho realizado na Clínica de Eco-Doppler Colorido, Curitiba, PR.

Artigo submetido em 14.09.05, aceito em 24.02.06. 
Apesar dos avanços no tratamento cirúrgico das varizes dos membros inferiores, a recidiva das varizes continua a ser relativamente freqüente. U ma das causas mais comuns de recidiva de varizes é a não detecção e correção do refluxo venoso nas veias safenas enas veias perfurantes no momento do primeiro tratamento ${ }^{1}$. Até recentemente, a pesquisa do refluxo venoso nas veias safenas era dificultada pela baixa sensibilidade do exame clínico e do ultra-som D oppler de ondas contínuas no segmento safeno-poplíteo ${ }^{2}$. Com o uso do ecoD oppler colorido, atingiu-seal ta sensibilidadeeespecificidade na pesquisa de refluxo nas veias safenas ${ }^{3,4}$.

0 uso do eco-D oppler colorido pré-operatório no tratamento cirúrgico de varizes dosmembrosinferiores é defendido por diversos autores, pois aumenta a possibilidade de detecção de refluxo venoso ede veias perfurantesinsuficientes, causas potenciais derecidiva precoce de varizes ${ }^{5-7}$. 0 eco-Doppler colorido permite a detecção de refluxo venoso najunção safeno-poplítea e na veia safena parva ${ }^{8}$, locais freqüentes de recidiva e de variaçõesanatômicas, quepodem dificultar o tratamento e provocar fal ha terapêutica? .

0 objetivo deste estudo é determinar a prevalência de refluxo venoso na veia safena parva em membros inferiores com varizes primárias não complicadas através do eco-Doppler colorido. A altura da junção safeno-poplítea também foi avaliada.

\section{M étodo}

$\mathrm{N}$ o período de 18 meses, foram avaliados retrospectivamente 1.953 pacientes com patologias venosas diversas demembrosinferiores, por meio do eco-D oppler colorido. D estes 1.953 pacientes, 1.631 eram portadores de varizes primárias não complicadas eforam selecionados para esta análise, sendo que 1.383 eram do sexo feminino $(84,79 \%)$ e $248(15,21 \%)$, do sexo masculino. Foram consideradas como não complicadas ${ }^{10}$ as varizes primárias das classes clínicas 0 e 1 da classificação de Porter ${ }^{11}$ (T abela 1) ou das classes clínicas 1 e 2, segundo a classificação CEAP12 (T abela 2).

A média deidadedospacientesfoi de $42,89( \pm 0,48)$ anos, variando de 13 a 85 anos. A média de idade entre as mulheres foi de $42,72( \pm 1,26)$ anos e, entre os homens, foi de $42,50( \pm 0,51)$ anos, variando de 13 a 85 e de 14 a 76 anos, respectivamente $(P=0,59)$.

D os 1.631 pacientes, 1.323 foram submetidos a exame bilateral e 308 a exame unilateral, totalizando 2.954 membros inferiores com varizes primárias não
Tabela 1 - Classificação de Porter ${ }^{11}$ para insuficiência ve nosa crônica dos membros inferiores

\begin{tabular}{ll}
\hline Classe 0 & Assintomáticos \\
Classe 1 & $\begin{array}{l}\text { Insuficiência venosa crônica leve, quando } \\
\text { há sintomasleves de desconforto, sensação } \\
\text { de membros inferiores pesados ou varizes } \\
\text { dolorosas, ou aindasinais de edema discre } \\
\text { to ao nível do tornozelo }\end{array}$ \\
& Insuficiência venosa crônica moderada, \\
Classe 2 & quando há edema mais importante do \\
& membro, alterações fibróticas subcutâneas \\
& ou hiperpigmentação de pele, sem que \\
& exista ulceração \\
Classe 3 & Insuficiência venosa crônica severa, quan- \\
& do existe ulceração ou lesão descamativa \\
préulcerosa, ou ainda edema severo de & todo o membro
\end{tabular}

Tabela 2 - Estratificação clínica (C) da classificação da insuficiência venosa crônica dosmembrosinferiores (CEAP) padronizada no sexto American Venous Forum (1995)

\begin{tabular}{ll}
\hline C0 & Sem sinais de doença venosa \\
C1 & T elangiectasias e/ou veias reticulares \\
C2 & V eias varicosas \\
C3 Edema sem alterações cutâneas \\
C4 Alterações tróficas de pele, como hiperpigmentação, \\
& eczema e lipodermatoesclerose \\
C5 Úlcera cicatrizada \\
C6 Ú lcera ativa
\end{tabular}

complicadas avaliados. Do total de 2.954 membros inferiores avaliados, 1.461 eram membros inferiores direitos e 1.493 eram esquerdos.

Além dos laudos de ultra-sonografia D oppler do sistema venoso dosmembrosinferiores, também foram analisadas as guias médicas de solicitação dos exames, bem como um pequeno questionário respondido pelos pacientes no dia do exame. $N$ esse questionário, além dos dados pessoais, constava o motivo para realização do exame, a realização ou não de cirurgia venosa prévia ea presença ou não decomplicações de doença venosa, como trombose venosa profunda, tromboflebite super- 
ficial eúlcera venosa, além da avaliação da presença de alterações cutâneas pelo examinador.

T odos os exames foram realizados com um apareIho System Five da General Eletric D iasonics U Itrasound ${ }^{\circledR}$, com transdutor linear de 7,5 a $10 \mathrm{M} \mathrm{H}$ z, com os pacientes em maca apropriada e em posição de T rendelenburg reverso com $30^{\circ}$ de inclinação, usando manobra de Valsalva e compressão e descompressão bruscas distais para avaliar o grau de refluxo ${ }^{13-15}$. Classificou-se como refluxo significativo quando o refluxo teve duração superior a 1 segundo.

Foram excluídos deste estudo os pacientes que apresentavam alterações cutâneas por hipertensão venosa crônica ou que apresentavam história de cirurgia venosa prévia e os exames realizados para pesquisa de trombose venosa ou compressão venosa.

A pós a análise dos dados, foram elaboradas tabelas de contingência. U m banco de dadosfoi montado e foram realizadas análises estatísticas paramétricas e não paramétricas dos dados. As comparações foram feitas utilizando o teste do qui-quadrado. 0 erro alfa foi estabelecido em $5 \%$ para rejeitar-se a hipótese nula. Para comparação entre médias, foi utilizada a análise de variância.

\section{Resultado}

D o total de 2.954 membros inferiores avaliados, 0 refluxo venoso em veia safena parva foi encontrado em 372 (12,59\%) (Figura 1).

D os 372 membros inferiores com refluxo em veia safena parva, em $23(6,18 \%)$ o refluxo era transmitido através da veia de $G$ iacomini.

A prevalência de refluxo em veia safena parva aumentou concomitantemente com a idade dos pacientes avaliados, sendo significativamente mais freqüente no grupo de pacientes com idade maior que 60 anos, conforme demonstra a Figura 2.

A presença de refluxo em veia safena parva foi pouco maior nos homens ( $14,08 \%)$ do que nas mulheres $(12,35 \%)$, porém sem diferença estatísticasignificativa $(P=0,32166)$.

A ocorrência de refluxo em veia safena parva foi maior no membro inferior esquerdo $(13,13 \%)$ do que no membro inferior direito $(12,05 \%)(P=0,037642)$.

A altura da junção safeno-poplítea nos membros com refluxo em veia safena parva foi encontrada em média 3,01 $( \pm 0,15) \mathrm{cm}$ acima da prega cutânea poplí-
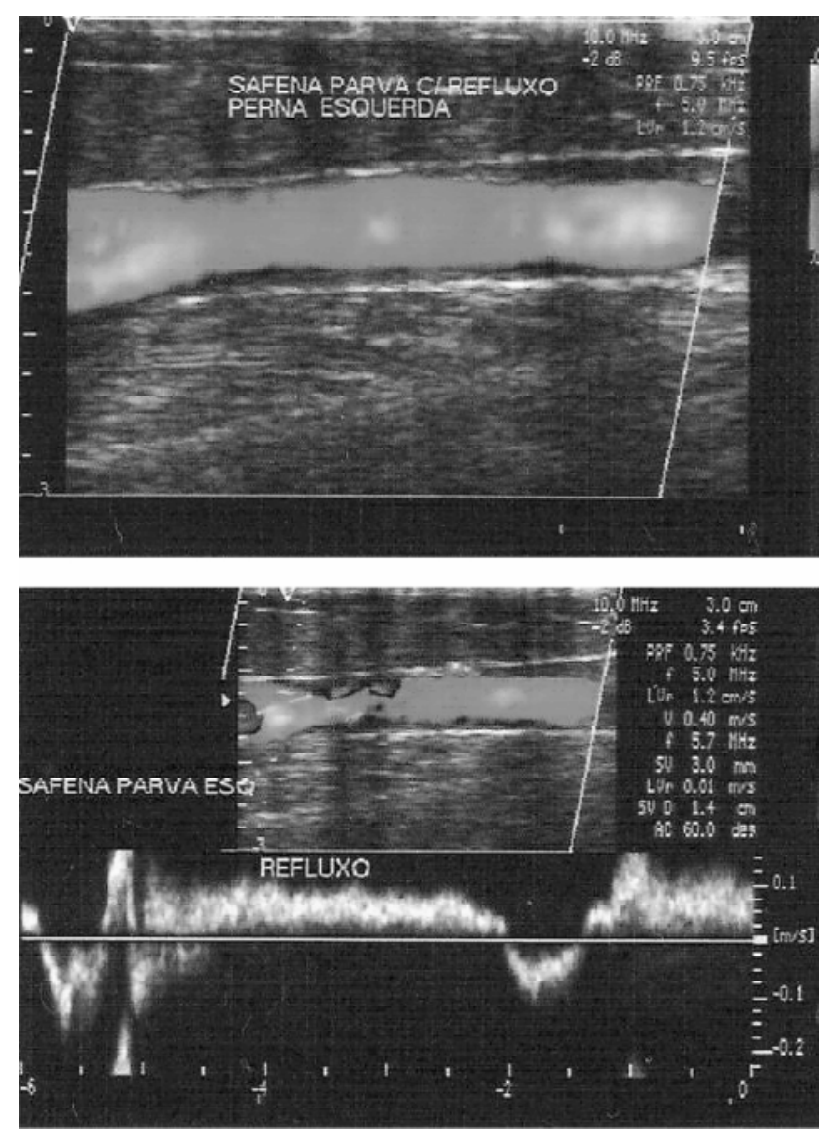

Figura 1 - Refluxo venoso em veia safena parvanaimagem ultra-sonográfica com Doppler colorido e dopplerfluxometria

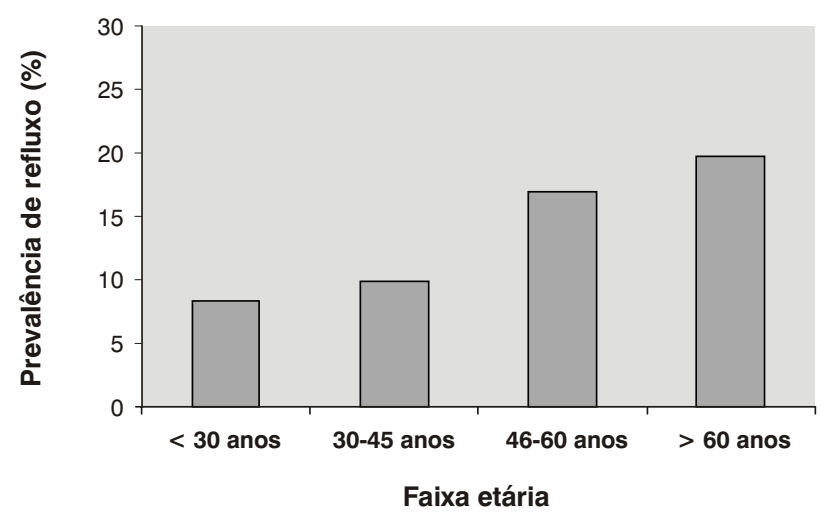

Figura 2 - Relação entre faixa etária e a prevalência de refluxo em veia safena parva $(P<0,000001)$

tea, variando entre 0 e $9 \mathrm{~cm}$ acima desta, enquanto que, nos pacientes sem refluxo em veia safena parva, ajunção safeno-poplíteafoi encontradaa 2,78 $( \pm 0,29) \mathrm{cm}$ acima da prega poplítea ( $P<0,0001)$. 


\section{D iscussão}

A presençade refluxo venoso em veias safenas, veias perfurantes e em sistema venoso profundo tem sido descrita amplamente na literatura como causa da formação de varizes, tanto primárias quanto secundárias à trombose venosa profunda ${ }^{5,16}$. Para detecção do refluxo venoso em sistema superficial, veias perfurantes $e$ sistema profundo, o melhor método é o eco-D oppler colorido, pois, além dealta sensibilidadee especificidade, evita os riscos da flebografia ${ }^{17-19}$.

A indicação do eco-D oppler colorido no pré-ope ratório de pacientes submetidos a tratamento cirúrgico de varizes dos membrosinferioreséamplamenteaceita. Diversos autores 0 indicam no pré-operatório, por aumentar a probabilidade de detecção de fatores que aumentam o risco darecidiva devarizes, como a presença de refluxo venoso em safenas e a presença de perfurantes insuficientes ${ }^{1,5,7,10,20-24}$, além de poder, em certos casos, evitar cirurgias radicais com retirada de veias safenas que poderiam ser utilizadas posteriormente para revascularização do miocárdio ou de membros inferiores ${ }^{23,25,26 .}$

Porém, há autores que defendem o uso seletivo do método, utilizando somente em casos em que há varizes no trajeto das safenas equando há presença de sintomas mais exuberantes, como dor e edema nos membrosinferiores, não se indicando o eco-D oppler colorido em pacientes com varizes distantes das veias tronculares (safenas), assintomáticas e sem sinais de insuficiência venosa mais grave (dor, edema ou alterações cutâneas) ${ }^{27}$.

Indicação absoluta defendida do eco-D oppler colorido no préoperatório de cirurgia de varizes é a presença de refluxo no exame com o D oppler de ondas contínuas em fossa poplítea e em casos de veia safena residual (em pacientescom recidivadevarizesjásubmetidos a safenectomia parcial) ${ }^{28,29}$.

A prevalência de refluxo em veia safena parva encontrada neste estudo foi de $12,59 \%$. D ependendo do método utilizado na detecção do refluxo, os resultados variam na literatura. Rivlin et al. encontraram $14 \%$ de refluxo em safena parva usando somente o exame clínico em pacientes com varizes primárias em qualquer classeclínica ${ }^{30}$. D arkeet al., analisando 98 membrosde pacientes com varizes primárias não complicadas, encontraram refluxo venoso em veia safena parva em $9,04 \%$ através do exame clínico e do D oppler de onda contínua e $21,42 \%$ usando o eco-D oppler colorido ${ }^{10}$. M cM ullin \& Smith encontraram 33\% de pacientes com refluxo em veia safena parva usando o ecoD oppler colorido, porém sem estratificação de classe clínica ${ }^{31}$. Vasdeskis et al.encontraram $19,14 \%$ de refluxo em veia safena parva analisando somente pacientes com varizes primárias sintomáticas e usando 0 eco-D oppler colorido 2 .

Evanset al. avaliaram umaamostrapopulacional de 1.566 indivíduos, na qual encontraram, usando o ecoD oppler colorido, a presença de refluxo venoso em veia safena parva em $17 \%$ dos indivíduos com doença venosa em qualquer classe clínica e em 2,3\% dos indivíduos sem qualquer sinal ou sintoma de doença venosa 32 .

$M$ yers et al.encontraram a presença de refluxo em veia safena parva em $18 \%$ dos pacientes com varizes primárias não complicadas (classes clínicas 0 e 1 de Porter e C 1 e C2 da classificação (EAP) e variando entre $26,50 \%$ a $62,04 \%$ dos pacientes com alterações cutâneas ou ulceração (classes clínicas 2 e 3 de Porer e $C 4, C 5$ ou $C 6$ da classificação CEAP). A prevalência de refluxo em veia safena parva encontrada por diversos autores usando a ultra-sonografia D oppler varia de 2,3 a $62,04 \%$, de acordo com a presença de doença venosa e sua classe clínica ${ }^{33}$.

A distribuição encontrada entre os pacientes incluídos neste estudo em relação ao sexo foi de $84,80 \%$ de mulheres e $15,20 \%$ de homens. A maioria dos autores descreve uma preponderância na prevalência de varizes em mulheres variando entre $55,93 \%$ e $84,86 \% 6,10,34$. Entretanto, quan do avaliamosa presença derefluxo em veia safena parva, a prevalência foi pouco maior nos homens $(14,08 \%)$ do que nas mulheres $(12,35 \%)$, porém sem diferença estatística significativa $(P=0,32166)$.

A presença de refluxo em veia safena parva encontrada neste estudo foi de $8,33 \%$ nos pacientes com idade até 30 anose de $19,78 \%$ nos pacientes com idade superior a 60 anos $(P<0,00001)$. A prevalência de refluxo venoso em veia safena parva demonstrada neste estudo aumentou proporcionalmentecom a faixa etária analisada, com diferença estatisticamente significativa.

Atualmente, o eco-D oppler colorido pré-operatório é considerado também importante para localização da junção safeno-poplítea ${ }^{8}$, já que esta é muito variável, e a avaliação clínica é eficaz em somente $50 \%$ dos casos ${ }^{35-37}$. A altura da junção safeno-poplítea, segundo Askar et al., é sempre ao nível ou logo acima da prega poplítea ${ }^{38}$. $\mathrm{H}$ aeger, através de um estudo anatômico, localizou a junção nos três pri- 
meiros cm acima da prega cutânea poplítea em $60 \%$ dos casos, encontrando-se todas entre $4 \mathrm{~cm}$ abaixo e $7 \mathrm{~cm}$ acima da prega poplítea ${ }^{39}$. No estudo realizado por Engel et al., utilizando o eco-D oppler colorido no pré-operatório de cirurgia de varizes, a junção safeno-poplítea localizava-se, em média, $3,4 \mathrm{~cm}$ acima da prega poplítea, estando entre 2 e $6 \mathrm{~cm}$ em $81,5 \%$ dos casos. A acurácia do método comparada aos achados intra-operatórios para a localização da junção foi de $93 \%{ }^{9}$. Al gunsautores descreveram que, em $8 \%$ dos casos, a veia safena parva tem sua desembocadura até 3 polegadas abaixo do joelho 40 .

N este estudo, a altura encontrada da junção safeno-poplítea foi de 2,95 $\pm 0,56 \mathrm{~cm}$ acima da prega cutânea poplítea, havendo diferença estatisticamente significativa nos membros com $(3,01 \pm 0,15)$ ou sem $(2,78 \pm 0,29)$ refluxo em veia safena parva $(P<0,0001)$. N ão houve diferença estatisticamente significativa comparando-se a localização da junção em membros direitos e esquerdos e os diferentes graus de refluxo em safena parva.

A imensa variação na altura da junção safenopoplítea tem origem na formação embriológica. A veia safena parva é a primeira veia superficial dos membros inferiores que se desenvolve no embrião. Inicialmente, termina na veia ilíaca interna, através das veias glúteas, depois estabelece comunicações com a veia safena magna e, finalmente, com o crescimento do membro inferior, desemboca na veia poplítea. Apresenta, portanto, três estágios de desenvolvimento, podendo ocasionar variações de terminação ou até mesmo comunicação com as veias com as quais mantinha ligação durante 0 período embrionário ${ }^{41}$.

U ma variante comum éa existência de uma comunicante entre as veias safenas magna e parva, chamada veia fêmoro-poplítea, veia cutâneo femoral posterior, ramo anastomótico superior ou veia de G iacomini. Essa veia comunica a veia safena parva em seu terço superior com a veia safena magna na junção do terço médio com o superior, mas pode se comunicar com a veia safena magna mais proximal, até próximo da junção safenofemoral ${ }^{42}$. D os 372 membros inferiores com refluxo em veia safena parva, em $23(6,18 \%)$ o refluxo era transmitido através da veia de G iacomini.

\section{C onclusões}

0 refluxo venoso na veia safena parva em pacientes com varizes não complicadas é relativamente comum
(12,59\%). Portanto, a avaliação da junção safenopoplítea nesses pacientes deve ser realizada de rotina.

A altura da junção safeno-poplítea em membros com refluxo venoso em safena parva é $3,01 \pm 0,15 \mathrm{~cm}$ acima da prega cutânea poplítea, variando de 0 a $9 \mathrm{~cm}$ acima da prega cutânea poplítea. N os membros inferiores que não apresentam refluxo na veia safena parva, a junção safeno-poplítea é mais baixa.

\section{R eferências}

1. Labropoulos N, T ouloupakis E, Giannoukas AD, Leon M, Katsamouris $A, N$ icolaides $A N$. Recurrent varicose veins: investigation of thepattern and extent of reflux with color flow duplex scanning. Surgery. 1996;119:406-9.

2. Vasdeskis SN, Clarke GH, Hobbs JT, Nicolaides AN . Evaluation of non-invasive and invasive methods in the assessment of short saphenous vein termination. $\mathrm{Br}$ J Surg. 1989;76:929-32.

3. N eglen P, Raju S. A comparison between descending phlebography and duplex Doppler investigation in the evaluation of reflux in chronic venousinsufficiency: achallenge to the phlebography as the "gold standard" . J Vasc Surg. 1992;16:687-93.

4. M oraes Filho D, EI H ozni J r RA, Diniz JAM, et al. U so do duplexultra-som no planejamento do tratamento cirúrgico de varizesdosmembrosinferiores. CirV asc Angiol. 1999;16:43-9.

5. Sarin S, Scurr JH, C oleridgeSmith PD . Assessment of stripping the long saphenous vein in the treatment of primary varicose veins. Br J Surg. 1992;79:889-93.

6. Luccas GC, N agase $Y, M$ eneses FH, et al. Cirurgia de varizes dos membros inferiores. Avaliação pré-operatória do sistema venoso com mapeamento duplex. Cir V asc Angiol. 1996;12: 15-20.

7. Engelhorn CA, Picheth FS, Castro Jr N, Dabul Jr NM, $\mathrm{G}$ omesC S. Estudo do sistemavenoso superficial com o duplex scan a cores. Cir Vasc Angiol. 1996;12:12-4.

8. Labropoulos N, Giannoukas AD, D elis K, et al. The impact of isolated lesser saphenous vein system incompetence on clinical signs and symptoms of chronic venous disease. J V asc Surg. 2000;32:954-60.

9. Engel $A F, D$ avies $G, K$ eeman JN . Preoperative localisation of the saphenopopliteal junction with duplex scanning. Eur J V asc Surg. 1991;5:507-9.

10. Darke SG, Vetrivel S, Foy DMA, Smith S, Baker S. A comparison of duplex scanning and continuous waveD oppler in the assessment of primary and uncomplicated varicose veins. Eur J V asc Endovasc Surg. 1997;14:457-61.

11. Porter JM, Rutherford RB, Clagett GP, et al. Reporting standards in venous disease: an update. Prepared by the Subcommitteeon Reporting Standardsin VenousD isease, Ad $\mathrm{H}$ oc Committee on Reporting Standards, Society for V ascular Surgery/N orth American Chapter, International Society for Cardiovascular Surgery. J V asc Surg. 1988;8:172-81.

12. Porter $M M, M$ oneta GL. Reporting standardsin venous disease. International consensus Committee on Chronic Venous D isease. J V asc Surg. 1995;21:635-45. 
13. M asudaEM , Kistner RL, Eklof B. Prospectivestudy of duplex scanning for venous reflux: comparison of $V$ alsalva and pneumatic cuff techniques in the reverse T rendelenburg and standing positions. J V asc Surg. 1994;20:711-20.

14. Benabou JE, M olnar L, C ardoso N eto FB, C erri G G, Leão PP. Avaliação por mapeamento duplex da junção safenofemoral em pacientes portadores de varizes recidivantes submetidos a prévia cirurgia radical de varizes. Cir V asc Angiol. 1996;12: 36-9.

15. BenabouJE. M apeamento duplex colorido dasveiasperiféricas. In: M olnar LJ , editor. U Itra-sonografiavascular. Rio deJ aneiro: Revinter; 2004. p. 117-55.

16. Labropoulos N, Leon M, G eroulakos G, V olteas N, C han P, $\mathrm{N}$ icolaides $\mathrm{AN}$. Venous hemodynamic abnormalities in patients with leg ulceration. Am J Surg. 1995;169:572-4.

17. M cl rvine AJ, Corbett CR, Aston N O, Sherriff EA, W iseman $P A$, Jamieson CW. The demonstration of saphenofemoral incompetence; Doppler ultrasound compared with standard clinical tests. Br J Surg. 1984;71:509-10.

18. Welch HJ, Faliakou EC, M CLaughlin RL, Umphrey SE, Belkin M, O'D onnell TF Jr. Comparison of descending phlebography, air plethysmography and duplex quantitative valveclosuretimein assessing deep venous reflux. J V asc Surg. 1992;16:913-9.

19. $N$ eglen $P$, Raju S. A rational approach to detection of significant reflux with duplexD oppler scanning and ai r plethysmography. J V asc Surg. 1993;17:590-5.

20. $H$ anrahan $L M$, Araki CT, Fisher JB, et al. Evaluation of the perforating veins of the lower extremity using high resolution duplex scanning. J Cardiovasc Surg. 1991;32:87-97.

21. Bradbury AW, Stonebridge PA, Ruckley CV, Beggs I. Recurrent varicose veins: correlation between preoperative clinical and hand-held D oppler ultrasonographic findings at surgery. Br J Surg. 1993;80:849-51.

22. Sarin S, Scurr JH, Coleridge Smith PD. Stripping of thelong saphenous vein in the treatment of primary varicose veins. $\mathrm{Br}$ J Surg. 1994;81:1455-8.

23. Luccas GC, Parente JBF, N agase $Y$, Lane JC. Preservação da veia safena magna em cirurgia de varizes: resultados tardios. Cir Vasc Angiol. 1995;11:15-8.

24. Sarquis AL. Avaliação pré e pós-operatória no tratamento cirúrgico conservador devarizestroncularescom o duplex scan a cores. Cir V asc Angiol. 1996;12:9-11.

25. Fonseca FP, Evangelista SSM, Sarquis AL. O tratamento cirúrgico ambulatorial e com anestesia local das varizes troncularesprimáriasdosmembrosinferiores, com preservação dassafenas - avaliação prée pós-operatória com o duplex scan e com a fotopletismografia. Cir V asc Angiol. 1996;12:19-22.

26. Rollo H A, Lastoria S, Yoshida W B, M oura R, M affei FH A. Cirurgia de varizes com preservação da veia safena magna avaliação pré e pós-operatória pelo mapeamento duplex resultados preliminares. Cir Vasc Angiol. 1996;12:63-8.

27. Labropoulos N, Leon M, N icolaides AN , Giannoukas AD, $V$ olteas N , Chan P. Superficial venousinsufficiency: correlation of anatomic extent of reflux with clinical symptoms and signs. J V asc Surg. 1994;20:953-8.
28. Campbell W B, H alim AS, A ertssen A, Ridler BM F, T hompson FJ , N iblett PG. Theplace of duplex scanning for varicoseveins end common venous problem. Ann R Coll Surg Engl. 1996;78:490-3.

29. W ali M A, Sheehan SJ, Colgan M P, M oore DJ, Shanik GD. Recurrent varicose veins. East Afr M ed J. 1998;75:188-91.

30. Rivlin S. Thesurgical cureof primary varicoseveins. BrJ Surg. 1975;62:913-7.

31. M cmullin GM, ColeridgeSmith PD . An evaluation ofD oppler ultrasound and photoplethysmography in theinvestigation of venous insufficiency. Aust N Z J Surg. 1992;62:270-5.

32. Evans CJ, Allan PL, Lee AJ, Bradbury AW, Ruckley CV, Fowkes $F G$. Prevalence of venous reflux in the general population on duplex scanning: the Edinburgh vein study. J V asc Surg. 1998;28:767-76.

33. M yersKA, Ziegenbein RW, Z eng $H, M$ atthewsPG. D uplex ultrasonography for chronic ven ous disease: patternsof venous reflux. J Vasc Surg. 1995;21:605-12.

34. Shami SK, Sarin S, CheatleT R, Scurr JH, Smith PD. V enous ulcer and the superficial venous system. J Vasc Surg. 1993;17:487-90.

35. M egret G. Étudecomparativeentrel'examecliniqueetl'exame D opplert acoustique de la saphène externe: a propos de 191 cas. Phelébologie. 1985;38:469-73.

36. Oliveira A, Vidal EA, França GJ, Toregiani J, Timi JRR, M oreiraR CR. Estudo davariação anatômica daterminação da veia safena parva por ecoDoppler colorido. J Vasc Br. 2004;3:223-30.

37. Sugrue M, Stanley S, Grouden M, Feeley M, M oore DJ, Shanik DG. Can pre-operative duplex scanning replace preoperative short saphenous venography as an aid to localizing the sapheno-popliteal junction? Phlebologie. 1988;41:722-5.

38. Askar 0. The short saphenous vein. J Cardiovasc Surg. 1963;4:126-37.

39. $\mathrm{H}$ aeger $\mathrm{K}$. The surgical anatomy of the sapheno-femoral and the sapheno-popliteal junctions. J Cardiovasc Surg. 1962;3:420-7.

40. M oosman D A, H artwell J $r$ SW. The surgical significance of thesubfascial course of thelesser saphenousvein. Surg $G$ yenecol O bstet. 1964;118:761-6.

41. Silva $A L$, Pelaquim AF, Borges $E$, Freire $M T G$, Rios $M$, Pereira LS. Vena saphena parva. Rev Bras Med. 1968;25: 389-92.

42. Burihan E. Estudo anatômico da veia safena parva [tese]. São Paulo: Escola Paulista de M edicina; 1972.

\section{Correspondência:}

Fabio Secchi

Rua Áustria 165, Jardim Europa

CEP 79826-400 - D ourados, MS

Tel.: (67) 421.0022/423.0334

E-mail: fsecchi@terra.com.br 